\title{
Fragment from an Anglo-Saxon Charm . Anonymous
}

Loud were they, loud as over the hill they rode, Were resolute as they rode over the land.

Shield thee now! that thou escape this malice.

Out little spear if ye herein be!

Stood, under linden wood under the light shield

While all the witch women-mihtigan wif-gathered their power, Sent spears ayelling.

I will send again to them, flying arrows to ward their advances.

Out little spear if ye herein be!

There sate the smith,

Struck the little sword,

Struck with hammer, mightily.

Out little spear if ye herein be!

Six smiths sat wrighting war spears.

Out, spear, lie not in, spear!

If herein be any iron at all

By witch work it to melting shall.

(ca. 1905) 